\title{
A Technique of Tool Manufacturing by changing the Polarity of EDM
}

\author{
Sandeep Lakra ${ }^{1}$, Rahul Charles Francis ${ }^{2}$ \\ ${ }^{1,2}$ Department of Mechanical Engineering, Vaugh Institute of Agricultural Engineering and Technology, Sam Higginbottom \\ University of Agriculture, Technology and Sciences, Prayagraj, Uttar Pradesh, India-211007 \\ ${ }^{1}$ Tony007sandeep@gmail.com \\ ${ }^{2}$ Rahul.francis@shiats.edu.in
}

\begin{abstract}
In today's modern era, cutting processes involves many vast technologies. EDM process is one of the advanced metal cutting processes. Since most of the tools are made from conventional processes in the lathe machine. This paper proposes a technique to manufacture some specific tools by changing the polarity in EDM. Changing the polarity means changing the path of the electron from tool to workpiece and workpiece to tool. This results more accurately and easily for manufacturing of complex-shaped tools.
\end{abstract}

Key Words: EDM, Electrode, Polarity, Solid Works

\section{INTRODUCTION}

Electric Discharge Machine (EDM) is a well-known and advanced process used to machine high precision dies, molds, and various other parts with complex features and high aspect ratio [1].

In the EDM process, an electric spark is used to cut the workpiece, which takes the shape inverse to that of the cutting tool or electrode.The electrode and the workpiece are both submerged in a dielectric liquid, which is generally light lubricating oil. A servomechanism keeps up a space of almost the thickness of a human hair between the electrode and the work, avoiding them from reaching each other. In EDM ram or sinker machining, generally soft graphite or metallic electrode can be utilized to cut solidified steel or even carbide. The EDM process produces a depth slightly bigger than the electrode because of the overcut [2]. Figure 1 shows the basic EDM process.

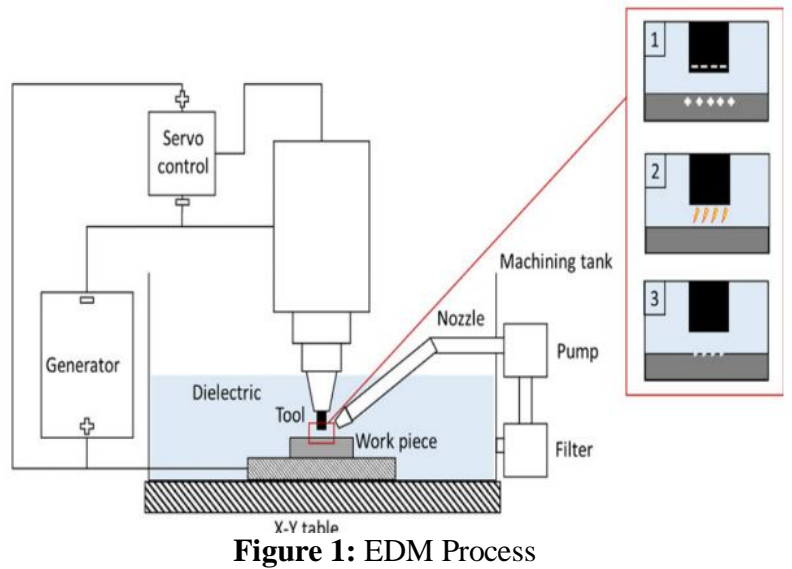

\subsection{Principle of EDM}

Electrical Discharge Machining (EDM) could be a controlled metal-removal process that's utilized to evacuate metal through electric spark erosion. In this process, an electric spark is utilized as the cutting tool to cut (dissolves) the workpiece to produce the finished part to the required shape. The metal-removal process is performed by applying a throbbing (ON/OFF) electrical charge of high-frequency current through the electrode to the workpiece. This evacuates (dissolves) very tiny pieces of metal from the workpiece at a controlled rate [3].

\subsection{EDM Process}

EDM spark erosion is the same as having an electrical short that burns a little gap in a piece of metal it contacts. For the EDM process, it is necessary for both the workpiece and the electrode to be conductors of electricity. The EDM process can be utilized in two different ways:

1. A pre-shaped or shaped electrode, usually made from graphite or copper, is formed to the shape of the cavity it is to reproduce. The shaped electrode is fed vertically down and the invert shape of the electrode is eroded (burned) into the strong workpiece.

2. A continuous traveling vertical electrode, the distance across of a little needle or less, is controlled by the 
computer to follow a programmed way to erode or cut a narrow slot through the workpiece to create the specified shape [3]. Figure 2 shows basic process of EDM

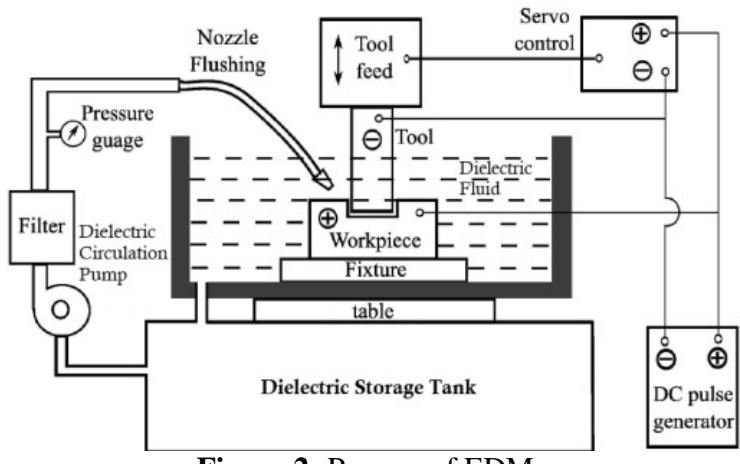

Figure 2: Process of EDM

\section{EXPERIMENTAL SETUP}

Experiments are done with a linear motor attached EDM machine to observe the change in the shape of the cylindrical electrode. The positive machine is equipped with an SVC circuit that can provide a rectangular pulse with a maximum frequency of $1 \mathrm{MHz}$.

A Copper cylindrical electrode having a $1 \mathrm{~mm}$ diameter is utilized to drill holes in a mild-steel workpiece. Positive polarity, pulse duration, pulse interval, material, and current peak are selected for investigation, Other discharge conditions are maintaining a discharge voltage of $16 \mathrm{~V}$, a servo voltage of $10 \mathrm{~V}$, the EDM oil working fluid, and an electrode bounce frequency of 30 times per minute. Side flushing is applied. Positive tests are performed at the surrounding temperature. Positive tool electrodes are made on-site by using a wire electrode discharge grinding process. Positive cross segments of the gaps were cut along their axis by a wire EDM machine. After the machining process, the gaps and the electrodes were analyzed through a metallographic microscope, SEM, and EDS. Figure 3 shows the basic setup of EDM.

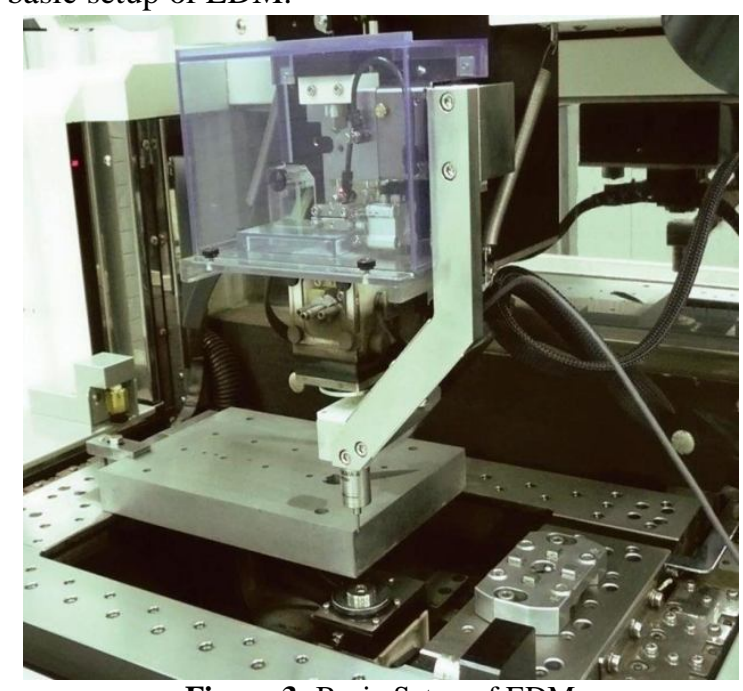

Figure 3: Basic Setup of EDM

\section{MATERIAL REMOVALMECHANISM (MRM)}

Material Removal Mechanism (MRM) is the process of transforming material elements between the workpiece and the electrode. The material elements get diffuse from the electrode to the workpiece and the same phenomenon can take place from the workpiece to the electrode. The transformation can occur in a solid, liquid, or gaseous state and finally alloyed with the contacting surface by undergoing a solid, liquid, or gaseous phase reaction. The state of sparking of MRM depends on the types of the eroded electrode and the work-piece elements together with disintegrated products of di-electric fluid [4].

\section{TOOL WEAR}

The tool wear process is similar to MRM as the tool and workpiece are considered as a set of electrodes in EDM Some valuable applications exploiting both the advantages and disadvantages of electrode wear have been developed.[5] presented a wear inhibitor carbon layer on the electrode surface by adjusting the settings of the method parameters earlier to typical EDM conditions. Even though the thickness of the carbon inhibitor layer made a noteworthy change on the TWR, it had little impact on the MRR [6]. Comparable tool wear compensation techniques have moreover been connected to micro-EDM, which is commonly executed in thin layers using basic cylindrical or tubular electrodes. [7]introduced a uniform tool wear machining method compensating the longitudinal instrument wear by applying an overlapping to to-and-fro machining movement. [8]initially evaluated the reduction of tool length based on pulse examination and subsequently compensated the tool wear by controlling the machining downward feeding movement in real-time. [9] determined the estimation of tool wear from the study of beat characteristics based on discharge voltage drop time. The different strategies of simulating the EDM process moreover give a great opportunity of understanding and compensating the tool wear. [10] created a geometrical simulation of EDM outlining the development of tool wear and part geometry. The simulation calculation is generally based on MRR, TWR, and spark hole. However, the recreation of discharge area and spark hole, which are dependent on the distribution of debris concentration, was detailed to surrender a more realistic representation of the startling phenomenon. [11]Other strategies include a switch simulation of EDM getting the shape of the electrode based on the required work-piece shape.[12] Figure 4 shows a two-dimensional model of the EDM machine process[13][16].Below Figure 4 shows two-dimensional model of EDM machining Process. 


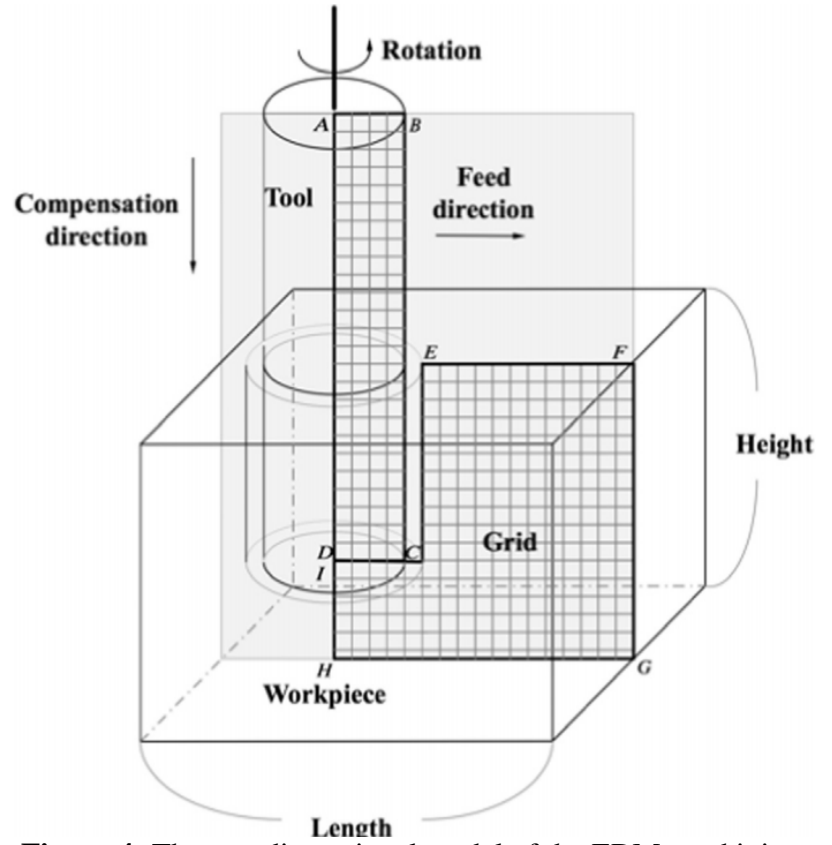

Figure 4: The two-dimensional model of the EDM machining process

\section{METHODS AND MECHANISM}

\subsection{Alter Process of Electrode Shape}

The dynamical changes of the electrode shape are considered. Since spark erosion happens on a workpiece within the working liquid and the shape of an electrode is exchanged onto the workpiece, the changes of the electrode shape over time are tracked by recording the cross areas of the different holes.

Pictures of the cross-section are made and watched every 2 minutes. There is some enlightenment of the final shape of the electrode, although these pictures are not continuous.

The shape changes can be seen. The convex shape comes into being within the 5th minute. Extending the preparing time, the convex height appears gradually and develops to a few degrees whereas remaining steady from the 9th minute to the 15th minute. Meanwhile, two electrode shapes and comparing cross-sections of the machined hole with two different machining times are presented. It can be seen that the shape of the electrode remains steady after 60 minutes of machining adjusting well with the convex tip shape at the hole.

The electrode is parameterized, as described, to helpfully analyze the comes about. D is the breadth of the electrode, $r$ and sr are the corner sweep and the spherical radius of the concave tip, respectively, $\delta$ represents the thickness of the skin layer, and $\mathrm{h}$ is the ring width of the end face.

Figure 5 shows the geometry of the model electrode

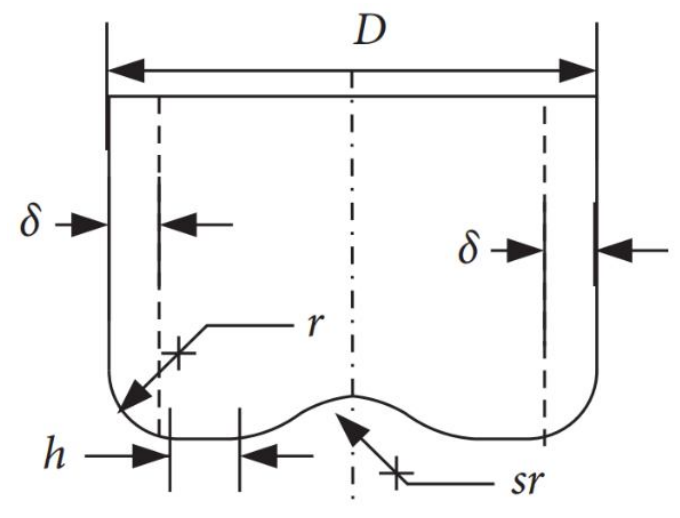

Figure 5: Geometry of the model electrode.

\subsection{Mechanism Analysis of the Electrode Shape Change.}

The electrode is usually connected to the negative terminal of the power control supply in EDM. As the electric field is maintained between the electrode and the workpiece, the free electrons on the electrode are exposed to electrostatic forces [13].

These electrons are then accelerated toward the workpiece through the oil and then collide with the electrons and dielectric particles. Such a collision may result in the ionization of a dielectric particle. In this way, more positive ions and electrons would be generated. This repeated process would intensify the concentration of electrons and ions in the dielectric medium. This concentration would be so high that the matter existing in that channel may be characterized as "plasma" [14].

The plasma produces a very large current as well as internal electric and magnetic fields [15]. As the electric and magnetic fields change extremely, the induced electric and magnetic fields produce a discharge circuit, which includes the tool electrode.

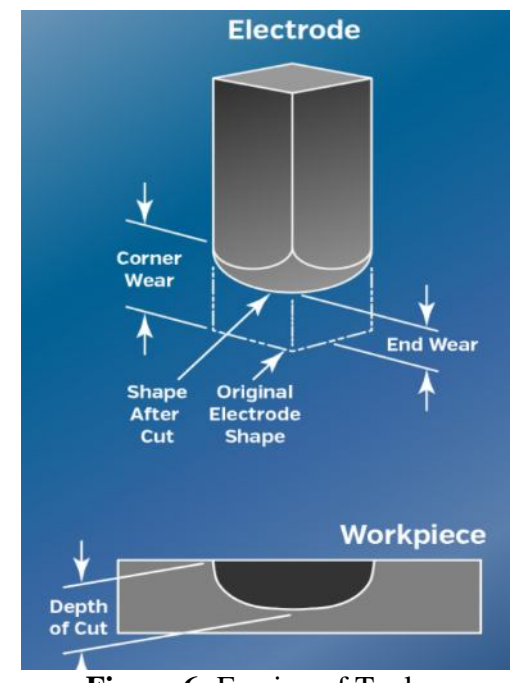

Figure 6: Erosion of Tool

the distance between the workpiece and the electrode is also small, which is beneficial to building up the discharge channel close to the central line. As the distance decreases, there's sufficient time for positive ions to reach the negative electrode, and as a result, the material at the center of the 
electrode is evacuated by accepting heat flux from ions. In the end, a concave surface is shaped on the bottom of the electrode. Above Figure 6 shows the erosion of Tool.

After the change in the polarity of the electrodes, the electrode is connected to the positive pole of the power supply. During the discharge breakdown stage, the electric field intensity distribution between the electrode and the workpiece isn't uniform due to the tip effect. According to the cathode field-induced electron discharge theory of metals, with the increase of the electric field strength, the free electrons on the work-piece will tunnel through a metal surface barrier by being compressed by a strong electric field so that the electrons are first released on the edge of the electrode face, which makes the wear at the edge of the electrode end increase

In any case, the number of electrons emitted by the workpiece in the central area corresponding to the electrode end surface is less, and the probability of discharge is less. Therefore, the material evacuation within the central area of the electrode and surface is less, and a convex surface will be formed at the bottom of the electrode. As the distance between the work-piece and the electrode decreases, the positive ions have sufficient time to reach the negative electrode, so the work-piece is bombarded by ions at the bottom of the gap. At long last, a concave surface is shaped at the bottom of the gap, and the curved surface shaped at the end of the electrode is much sharper. If the positive electrode is connected to the electrode in EDM, the electrode will be severely worn by the huge amount of electron bombardment, while the processing speed of the workpiece is relatively slow.

\section{DESIGN OF EXPERIMENT COMPONENT}

Design of Components of the whole setup of the experiment had been done on SolidWorks 2016 software.

\subsection{Design of Tool}

Figure7 shows a cavity made on a material that is used as a Tool after changing the polarity.

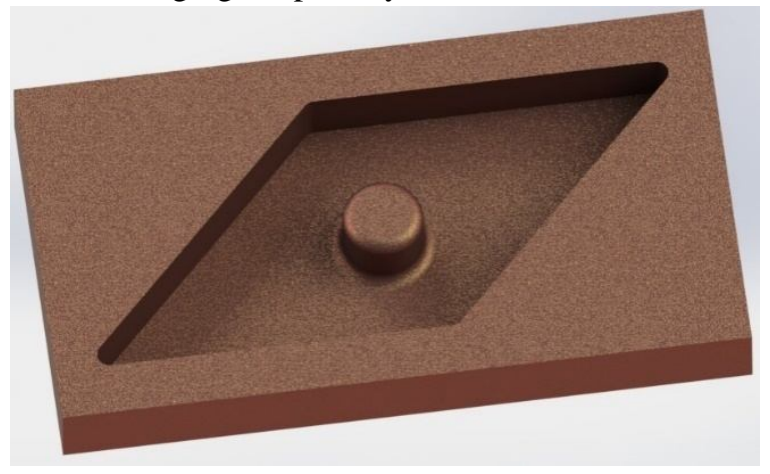

Figure 7:Material used as Tool

\subsection{Design of Workpiece}

Figure 8 shows the final image of the workpiece which is made by the above-shown Tool. The workpiece owns the exact shape and size of the cavity (Tool).

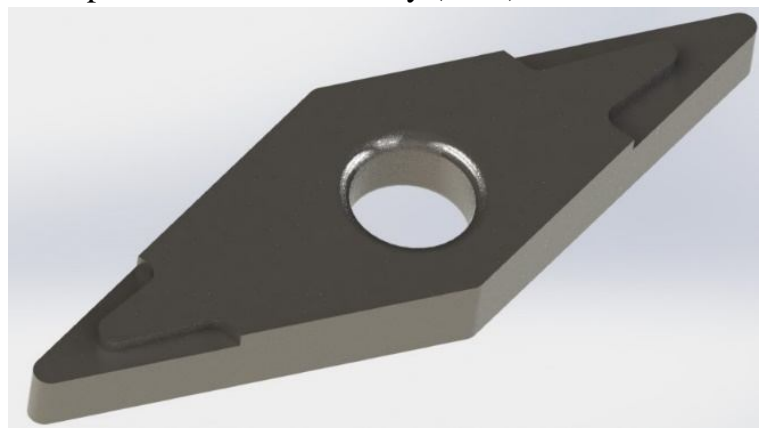

Figure 8: Material used as Workpiece

\section{CONCLUSION}

This section describes the conclusion of the proposed research work. As the EDM process has many essential advantages in today's world but this paper concludes a basic study of technique to manufacture some specific tools which are made by the conventional methods so far but after proposing this technique there will be an accurate and easy way to manufacture complex-shaped tools too as per the design.

\section{REFERENCES}

1 X. Li, Y. Wang, Y. Liu and . F. Zhao, "Research on Shape Changes in Cylinder Electrodes Incident to Micro-EDM," Advances in Materials Science and Engineering, pp. 1-11, 2019.

2 H. Marashi, A. Sarhan, I. Maher and M. Sayuti, "Techniques to Improve EDM Capabilities: A Review," Comprehensive Materials Finishing, vol. 1, pp. 171-202, 2017.

3 S. Krar, "Electrical Discharge Machining," [Online]. Available: https//www.automationmag.com.

4 N. Malhotra, S. Rani, and H. Singh, "Improvements in Performance of EDM-A Review," IEEE, pp. 599-603, 2008.

5 . J. Marafona and, C. Wykes, "A new method of optimising material removal rate using EDM with copper-tungsten electrodes," International Journalof Machine Tools Manufacturing, vol. 40, no. 2, pp. 153164, 2000.

6 M. Kunieda and H. Muto, Annals. CIRP, vol. 49, no. 1, p. Annals. CIRP, 2000.

7 Y. Z. Yu, T. Masuzawa and M. Fujino, "Micro-EDM for three-dimensional cavities-development of uniform wear method," Ann. CIRP, vol. 47, no. 1, pp. 169-172, 1998.

8 P. Bleys, P. J. Kruth, B. Lauwers, A. Aryd, R. Delpretti, 
and C. Tricarico, "Real-time tool wear compensation in milling EDM," Ann. CIRP, vol. 51, no. 2, pp. 157160, 2002.

9 D. Dauw and R. Snoeys, "On the derivation and application of a real-time tool wear sensor in EDM," Annals. CIRP, vol. 35, no. 1, pp. 111-116, 1986.

10 D. Dauw, "Geometrical simulation of the EDM diesinking process," Annals. CIRP, vol. 37, no. 1, pp. 191196, 1988.

11 M. Kunieda and M. Kiyohara, "Simulation of diesinking EDM by discharge location searching algorithm," Int. J.Electr. Machining, pp. 79-85, 1998.

12 M. Kunieda, W. Kowaguchi and T. Takita, "Reverse simulation of die-sinking EDM," Annal. CIRP, vol. 48, no. 1, pp. 115-118, 1999.

13 S. S. Mujumdar, D. Curreli, S. G. Kapoor and D. Ruzic, "A model of micro electro-discharge machining plasma discharge in deionized water," Journal of Manufacturing Science and Engineering, vol. 136, no. 3, 2014.

14 Y. V. Serdyuk and S. M. Gubanski, "Computer modeling of interaction of gas discharge plasma with solid dielectric barriers," IEEE Transactions on Dielectrics and Electrical Insulation, vol. 12, no. 4, pp. 725-735, 2005.

15 R. Vogelsang, B. Fruth, T. Farr, and K. Frohlich, "Detection of electrical tree propagation by partialdischarge measurement," European Transactions on Electrical Power, vol. 15, no. 3, pp. 271-284, 2005.

16 J. Pei, L. Zhang, J. Du, X. Zhuang, Z. Zhou, S. Wu, and Y. Zhu, "A model of tool wear in electrical discharge machining process based onelectromagnetic theory," International Journal of Machine Tools \& Manufacture, pp. 31-41, 2017. 